\title{
PDE-BASED AND SOLUTION-DEPENDENT PARAMETERIZATION FOR ISOGEOMETRIC ANALYSIS
}

\author{
Zulfiqar Ali ${ }^{1}$, Weiyin $\mathrm{Ma}^{1}$ \\ ${ }^{1}$ Department of Mechanical Engineering, City University of Hong Kong, Hong Kong, China \\ zzali2-c@my.cityu.edu.hk,mewma@ cityu.edu.hk
}

Key words: Isogeometric analysis, Isogeometric collocation method, Partial differential equations, NURBS, planar parameterization

\begin{abstract}
This paper presents some results for PDE-based and solution-dependent parameterization of computational domains for isogeometric analysis (IGA) using non-uniform rational B-splines (NURBS). The final parameterization is produced based on the solution of a partial differential equation (PDE) that is solved using isogeometric collocation method (IGA-C) with Dirichlet boundary condition being the input boundary of the final desired computational domain for IGA, namely the IGA-C-PDE method for domain parameterization. The theory of PDE guarantees that the mapping between physical and transformed region will be one-to-one. In addition, we also apply intuitive position and ratio constraints while solving the PDE to achieve solution-dependent parameterization. While one may use any general PDE with any constraint, the PDEs and additional constraints selected in our case are such that the resulting solution can be efficiently solved through a system of linear equations with or without additional linear constraints. This approach is different from typical existing parameterization methods in IGA that are often solved through an expensive nonlinear optimization processes. The results show that the proposed method can efficiently produce satisfactory analysis-suitable parameterization.
\end{abstract}

\section{Introduction}

Isogeometric collocation method (IGA-C) was introduced as an alternative approach to conduct engineering analysis without the need of efficient integration rules, as required in the standard isogeometric Galerkin methods (IGA-G). The main idea of IGA-C rests on the discretization of the governing partial differential equations in strong form at designated collocation positions which leads to the use of reduced number of evaluations for setting up the system of equations to only one per degree of freedom $[1,2]$. The advantage of low computational cost with promising results attracts many researchers across the globe to use IGA-C method in various applications including phase-field modeling [3], contact problems [4] and nonlinear elasticity [4]. This paper presents some results for solution-dependent parameterization of computation domains using a PDE-based IGA-C method [5] with intuitive position and ratio constraints for IGA, which corresponds to the mesh generation process in Finite Element Analysis (FEA), and has a key impact on analysis result and efficiency. One of the significant challenges towards IGA is the construction of an analysis suitable parameterization from a given CAD boundary representation [1]. Generally in IGA, the parameterization of the computational domain is determined by the representation of the computational domain whose parameters are degrees of the basis functions, knot vectors and control points of the B-spline or NURBS model. For 2D and 3D IGA problems, the knot vectors and the 
degrees of the computational domain are often determined by the given boundary curves/surfaces. The quality of the parameterization of the computational domain is determined by the knots and the distribution of the control points. A basic requirement of the resulting parameterization for IGA as discussed in [6] is that an analysis-suitable parameterization of the computational domain should have no selfintersections i.e. the mapping from the parametric domain to physical domain should be injective. Also, the iso-parametric elements should be as far as possible uniform and orthogonal. Most parameterization methods in the literature are based on the minimization of a quality functional. Commonly used quality functional include area orthogonality, Liao, Winslow , uniformity and harmonic energy [6]. One of the latest contribution for efficient parameterization of planar domain for IGA is reported in [7], where the domain is mapped to an equivalent convex domain using harmonic functions. Similarly, an IGA-suitable planar B-spline parameterization of the computational domain with high genus and more complex boundary curves is reported in [8]. Xu et al. also presented various approaches for the parameterization of the computational domain in IGA such as constrained optimization methods and shape optimization methods for generating analysis aware parameterization with a limitation that the solution is problem dependent $[9,10]$. Martin et al. proposed a method to fit a genus-0 triangular mesh by B-spline volume parameterization, based on discrete volumetric harmonic functions that can be used to build computational domains for 3D IGA problems [11]. A general framework for constructing planar parameterization has also been introduced in IGA using linear and non-linear constraints [12]. Falini et al. also proposed planar domain parameterization using THB-splines [13]. However, if we look in FEA, a commonly used method for mesh generation is based on solutions of partial differential equations (PDEs) [14]. The solution of an elliptical PDEs with Dirichlet boundary conditions on all boundaries produces the grid points / mesh

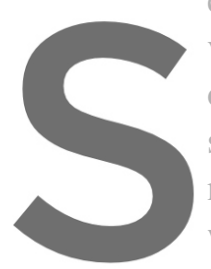
vertices. The theory of PI one to one. There are sever solution. However, a limin near convex (concave) we developed a framewor
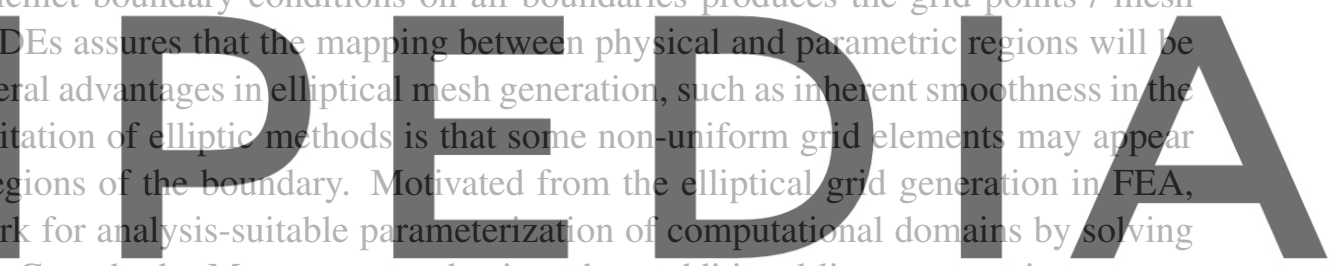

a PDE system using IGA-C methods. Moreover, we also introduce additional linear constraints to pro-

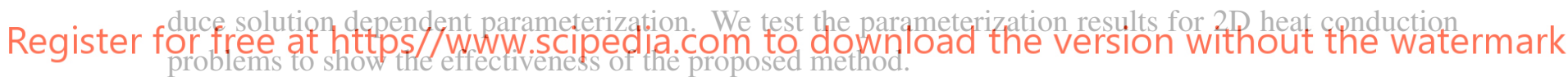

\section{Isogeometric collocation methods for solving PDEs}

\subsection{B-splines and NURBS}

Non-Uniform Rational B-spline (NURBS) are the basis functions widely used to represent the geometry for analysis in IGA. A B-spline of polynomial degree $p$ or order $k=p+1$ is defined by a knot vector $\Xi=\left\{\xi_{1}, \xi_{2}, \cdots, \xi_{n+k}\right\}$, with $n$ the number of basis functions. B-spline basis functions $\left\{\hat{N}_{i}(\xi)\right\}_{i=1}^{n}$ can be uniquely defined in a recursive manner as:

$$
\begin{gathered}
\hat{N}_{i, 1}(\xi)= \begin{cases}1, & \xi_{i} \leq \xi<\xi_{i+1} \\
0, & \text { otherwise }\end{cases} \\
\hat{N}_{i, k}(\xi)=\frac{\xi-\xi_{i}}{\xi_{i+k+1}-\xi_{i}} \hat{N}_{i, k-1}(\xi)+\frac{\xi_{i+k}-\xi}{\xi_{i+k}-\xi_{i+1}} \hat{N}_{i+1, k-1}(\xi), \quad k>1 .
\end{gathered}
$$


Given two univariate B-spline basis $\left\{\hat{N}_{i}(\xi)\right\}_{i=1}^{n_{\xi}}$ and $\left\{\hat{N}_{i}(\eta)\right\}_{i=1}^{n_{\eta}}$ respectively of order $k_{\xi}$ and $k_{\eta}$, and associated to the two knot vectors $\Xi_{\xi}=\left\{\xi_{1}, \xi_{2}, \cdots, \xi_{n_{\xi}+k_{\xi}}\right\} \Xi_{\eta}=\left\{\eta_{1}, \eta_{2}, \cdots, \eta_{n_{\eta}+k_{\eta}}\right\}$, a bivariate NURBS basis function is defined as:

$$
\hat{R}_{i, j}(\xi, \eta)=\frac{w_{i j} \hat{N}_{i, k_{\xi}}(\xi) \hat{N}_{j, k_{\eta}}(\eta)}{\sum_{i=1}^{n_{\xi}} \sum_{j=1}^{n_{\eta}} w_{i j} \hat{N}_{i, k_{\xi}}(\xi) \hat{N}_{j, k_{\eta}}(\eta)} \quad \forall(\xi, \eta) \in \hat{\Omega}
$$

where $w_{i j}$ is the weight associated to the basis function $\hat{R}_{i, j}$. NURBS surfaces $\mathbf{S}(\xi, \eta)$ are defined by:

$$
\mathbf{S}(\xi, \eta)=\sum_{i=1}^{n_{\xi}} \sum_{j=1}^{n_{\eta}} \mathrm{S}_{i j} \hat{R}_{i, j}(\xi, \eta)=\sum_{l=1}^{n} \mathrm{~S}_{l} \hat{R}_{l}(\xi, \eta), \quad(\xi, \eta) \in\left[\xi_{k_{\xi}}, \xi_{n_{\xi+1}}\right] \times\left[\xi_{k_{\eta}}, \xi_{n_{\eta+1}}\right],
$$

where $\left\{\mathrm{S}_{l}\right\}_{l=1}^{n}=\left\{\left\{\mathrm{S}_{i j}\right\}_{i=1}^{n_{\xi}}\right\}_{j=1}^{n_{\eta}}$ represent the set of $n=n_{\xi} \times n_{\eta}$ control points for the geometry. Using the isogeometric approach, the solution domain $\mathbf{u}(\xi, \eta)$ is approximated by the same basis functions that represent the geometry such that:

$$
\mathbf{u}(\xi, \eta)=\sum_{i=1}^{n_{\xi}} \sum_{j=1}^{n_{\eta}} \mathrm{u}_{i j} \hat{R}_{i, j}(\xi, \eta)=\sum_{l=1}^{n} \mathrm{u}_{l} \hat{R}_{l}(\xi, \eta)
$$

where $\mathrm{u}_{l}$ is the solution control points corresponding to the geometry control points $\mathrm{S}_{l}$.
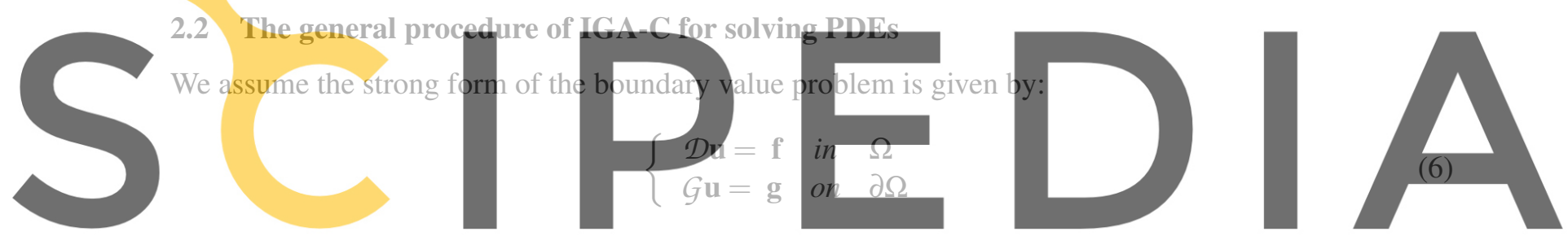

where $\mathbf{u}: \Omega \rightarrow \mathbb{R}$ represents the unknown solution, $\mathcal{D}$ is a linear differential operator, $G$ is a vector opera-

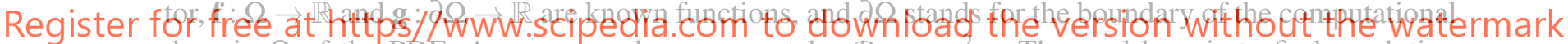
domain $\Omega$ of the PDE. As an example, one can take $\mathcal{D} \mathbf{u}=\nabla^{2} \mathbf{u}$. The goal here is to find a solution

u $: \Omega \rightarrow \mathbb{R}$ meeting the strong form of BVP. To solve this problem by the collocation method, two sets of collocation points need to be considered, a set $\bar{S}_{i}^{\text {int }}, i=1, \ldots, m^{i n t}$ in the interior of $\Omega$ and a set $\bar{S}_{i}^{b n d}$, $i=1, \ldots, m^{b n d}$ on $\partial \Omega$, where $m^{i n t}$ and $m^{\text {bnd }}$ are the number of collocation points chosen in the interior and on the boundary, respectively. Than, the collocation solution shown in Eq. 5 is required to satisfy:

$$
\left\{\begin{array}{rcl}
\mathcal{D} \mathbf{u}\left(\bar{S}_{i}^{\text {int }}\right)=\mathbf{f}\left(\bar{S}_{i}^{\text {int }}\right) & \text { in } \Omega & , i=1, \ldots, m^{\text {int }} \\
\mathcal{G} \mathbf{u}()\left(\bar{S}_{i}^{\text {bnd }}\right)=\mathbf{g}\left(\bar{S}_{i}^{\text {bnd }}\right) & \text { on } \partial \Omega & , i=1, \ldots, m^{\text {bnd }}
\end{array}\right.
$$

This will leads to a system of linear equations with linear constraints

$$
\mathbf{K u}=\mathbf{F}
$$

In Eq. (8), $\mathbf{K} \in \mathbb{R}^{(m \times n)}$ is a stiffness matrix, $\mathbf{u} \in \mathbb{R}^{(n \times 1)}$ is the solution vector, and $\mathbf{F} \in \mathbb{R}^{(m \times 1)}$ load vector acting upon the system, The total number of equations $m$ is usually equal to the number of unknowns $n$ and a unique solution for $\mathbf{u}$ can be found. If the total number of equations is more than the number of unknowns, i.e. $m>n$, Eq. (8) can then be solved using a constrained linear least-squares algorithm 
with exact satisfaction of desired boundary constraints similar to that reported in [15]. The traditional IGA-C uses the so-called "Greville abscissa" to define the collocation points that are obtained via knots averaging. For a given knot vector $\Xi_{\xi}$ and $\Xi_{\eta}$ the associated Greville points can be calculated as:

$$
\begin{array}{cc}
\bar{\xi}_{i}=\frac{\xi_{i+1}+\xi_{i+2}+\cdots+\xi_{i+p}}{p} & \text { for } i=1, \cdots, n_{\xi}, \\
\bar{\eta}_{j}=\frac{\eta_{j+1}+\eta_{j+2}+\cdots+\eta_{j+q}}{q} & \text { for } j=1, \cdots, n_{\eta},
\end{array}
$$

The collocation points $\chi_{i j} \in \hat{\Omega}$ as a tensor product structure can be defined as:

$$
\bar{\chi}_{i j}=\left(\bar{\xi}_{i}, \bar{\eta}_{j}\right), i=1, \cdots, m_{\xi} ; j=1, \cdots, m_{\eta},
$$

Collocation points in physical domain can be defined using a push-forward operator as:

$$
\overline{\mathrm{S}}_{i j}=\mathrm{S}\left(\bar{\chi}_{i j}\right), i=1, \cdots, m_{\xi} ; j=1, \cdots, m_{\eta} .
$$

\section{IGA-C with additional constrints for PDE-based parameterization}

In this section, we briefly highlight the procedure to produce 2D-parameterization of a desired computational domain $\left(\Omega_{1}\right)$ via solving a Laplace PDE on a simple initial computational domain $\left(\Omega_{0}\right)$. Four arbitrary boundary curves $\mathbf{X}=\bigcup_{i=1}^{4} \mathbf{X}_{i}$ is imposed as a Dirichlet boundary conditions on a $\left(\Omega_{0}\right)$ domain

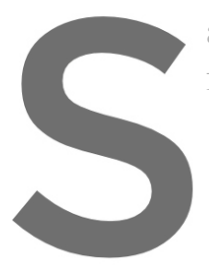
and Laplace PDE is solve form along with Dirichlet
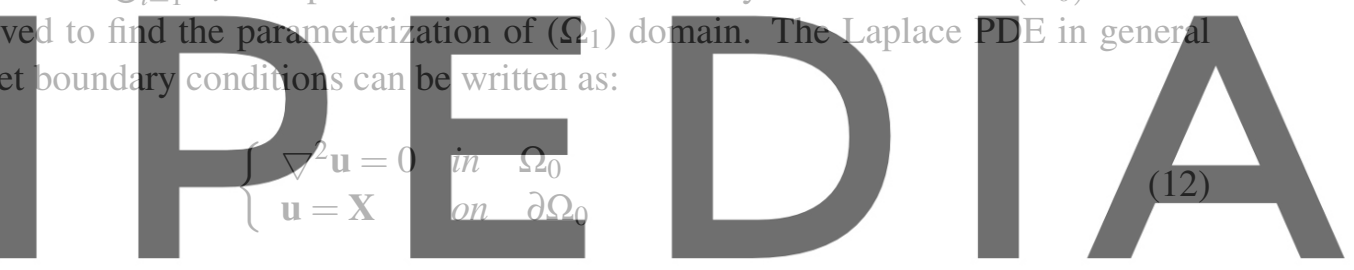

Register for free at heteps/Awinw.scipedia.com to download the version without the watermark

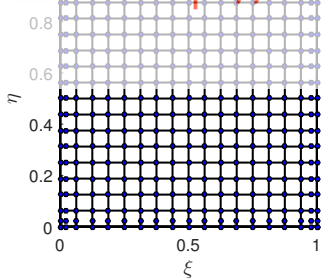

(a) $\hat{\Omega}$

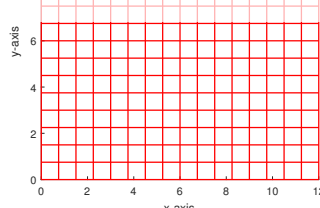

(b) $\Omega_{0}$

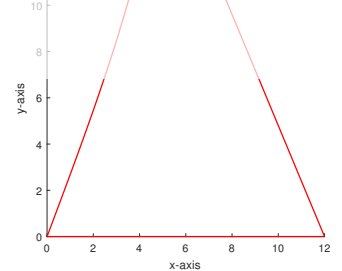

(c) $\partial \Omega_{1}$

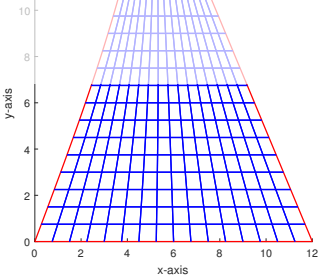

(d) $\Omega_{1}$

Figure 1: IGA-C-PDE based parameterization process: (a) the parametric domain for IGA-C; (b) an initial computational domain; (c) input boundary of a desired computational domain; and (d) the resulting parameterization of the desired computational domain / physical domain

While the target application of the resulting parameterization will be for IGA, the solution of PDE, i.e., the desired parameterization of $\Omega_{1}$ domain for IGA, is also produced using the IGA-C method with given initial parameterization of a simple $\Omega_{0}$ domain. To solve the PDE as shown of Eq. (12) by collocation method, a set of collocation points $\left\{\bar{\chi}_{i j}\right\}=\left\{\left(\bar{\xi}_{i}, \bar{\eta}_{j}\right)\right\}$, for $i=1, \cdots, n_{\xi}, j=1, \cdots, n_{\eta}$, in parametric 
domain are defined as Greville abscissa. $\bar{\chi}_{i j}$ can be further represented in physical domain as $\overline{\mathbf{S}}_{i j}$ via mapping as shown in Eq. (11). After introducing all collocation points $\bar{\chi}_{i j}=\left(\bar{\xi}_{i}, \bar{\eta}_{j}\right)$ or $\bar{S}_{i j}=\mathbf{S}\left(\bar{\chi}_{i j}\right)$ in Eq. (12), we obtain the final system of equations as:

$$
\begin{cases}\frac{\partial^{2} \mathbf{u}\left(\overline{\mathrm{S}}_{i j}\right)}{\partial x^{2}}+\frac{\partial^{2} \mathbf{u}\left(\overline{\mathrm{S}}_{i j}\right)}{\partial y^{2}}=0, & \text { for } i=2, \cdots, n_{\xi}-1 ; j=2, \cdots, n_{\eta}-1 \\ \mathbf{u}\left(\bar{\xi}_{i}, \bar{\eta}_{1}\right)=\mathrm{X}_{1}\left(\bar{\xi}_{i}\right), \mathbf{u}\left(\bar{\xi}_{i}, \bar{\eta}_{n_{\eta}}\right)=\mathrm{X}_{3}\left(\bar{\xi}_{i}\right), & \text { for } i=1, \cdots, n_{\xi} \\ \mathbf{u}\left(\bar{\xi}_{1}, \bar{\eta}_{j}\right)=\mathrm{X}_{2}\left(\bar{\eta}_{j}\right), \mathbf{u}\left(\bar{\xi}_{n_{\xi}}, \bar{\eta}_{j}\right)=\mathrm{X}_{4}\left(\bar{\eta}_{j}\right), & \text { for } j=2, \cdots, n_{\eta}-1\end{cases}
$$

which leads to a system of linear equations with linear constraints in the same form as shown in Eq. (8) with $m=n$. We will solve two Laplace PDE with respect to $\mathrm{x}$ and $\mathrm{y}$ coordinates for finding parameterization.

In this study, we also introduce additional linear constraints while solving the Laplace PDE in order to produce good parameterization with well-behaved Jacobian distribution. In the final solution of the constrained PDE, we ensure exact satisfaction for both the boundary constraints and additional constraints, while other parameters of the parameterization, mainly the interior control vertices, are further solved in the least squares sense. Hence, we are solving linear system of equations using constrained linear least squares methods in which the additional constraints are strictly satisfied as show in Eq 14.

$$
\left\{\begin{array}{l}
\mathrm{Ku}=\mathrm{F} \\
\text { s.t. } \mathrm{Cu}=\mathrm{d}
\end{array}\right.
$$

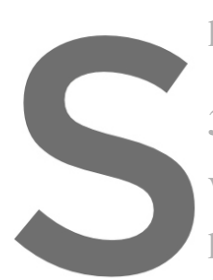

here $\mathrm{C}$ is the constrained matrix and $\mathbf{d}$ is a virtual loading veetor

3.1 Position constraints at selected locations

When solving the PDE, we can apply additional po

positions of the computational domain, for directly fos

parameterization, or for fixing both positions of

system shown in Eq. 12 is extended as Eq. 15:

Register for free at https//www.scipedia.com to download the version without the watermark

$$
\begin{cases}\nabla^{2} \mathrm{u}=0 & \text { in } \Omega_{0} \\ \mathrm{u}=\mathbf{X} & \text { on } \partial \Omega_{0} \\ \mathbf{u}(\xi, \eta)=\mathbf{X}(\xi, \eta) & \text { in } \Omega_{0} \\ \mathbf{u}_{I J}=\hat{\mathbf{u}}_{I J} & \text { for } 1<I<n_{\xi} ; 1<J<n_{\eta}\end{cases}
$$

\subsection{A ratio constraint for controlling local degrees of freedom}

The ratio among selected control points can also be used as a constraint to control the local degrees of freedom (DOFs), i.e., density of DOFs per unit dimension, for final parameterization. The PDE with respective ratio constraints for the basic topological structure is given in the following equation and is shown in Fig. 2(a),

$$
\begin{cases}\nabla^{2} \mathbf{u}=0 & \text { in } \Omega_{0} \\ \mathbf{u}=\mathbf{X} & \text { on } \partial \Omega_{0} \\ \left(V_{1}-V_{0}\right)=\lambda\left(V_{2}-V_{0}\right) & \forall V_{0} \in \Omega_{0}\end{cases}
$$

Here, $\left\{V_{0}, V_{1}, V_{2}\right\}$ are topologically aligned control points, either horizontally, vertically or diagonally aligned with respect to position $V_{0}$. Some of possible schemes or topological structures are shown in 


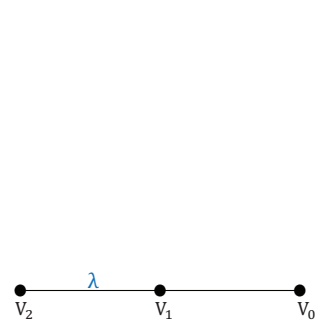

(a)

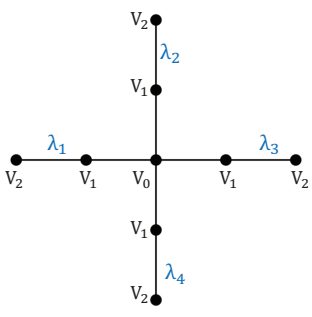

(b)

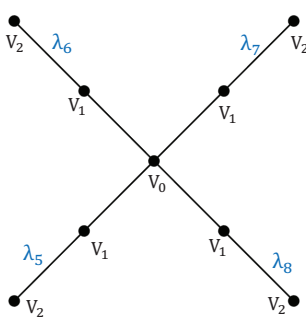

(c)

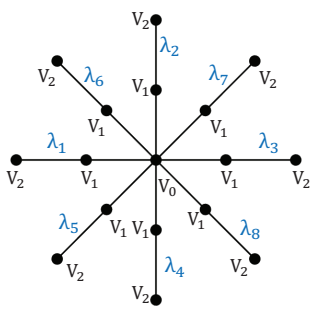

(d)

Figure 2: Basic schemes to control the ratio between control points. (a) basic Topology (b) scheme-I (c) scheme-II (d) scheme-III

Fig 2. The value of $\lambda$ is defined as the distance ratio among these control points as shown in Eq. 16 . When $\lambda<0.5, \lambda=0.5$ or $\lambda>0.5$, one achieves local converging, uniform or diverging distribution of the control points, respectively. Or in other words, the local DOFs will increase with $\lambda<0.5$ and the local DOFs will decrease with $\lambda>0.5$, which uniform distribution of control points is achieved when $\lambda=0.5$. The ratio constraint can be applied at positions of either control points or domain points.

4 Numerical results for parameterization with position and ratio constraints

\subsection{An example with fixed position constraint}

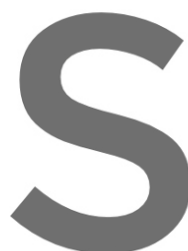

The first example is for pro

The parametric domain focomputational domain $x$

3. In this case, the simple

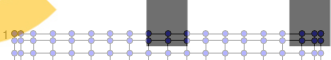

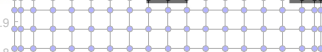

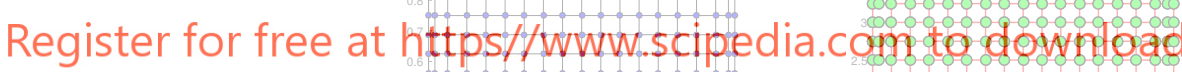

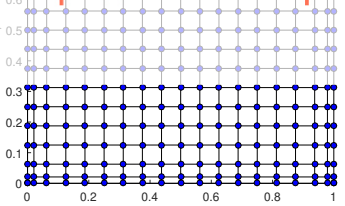

(a) $\hat{\Omega}$

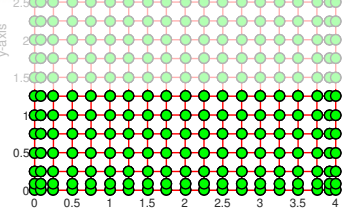

(b) $\Omega_{0}$
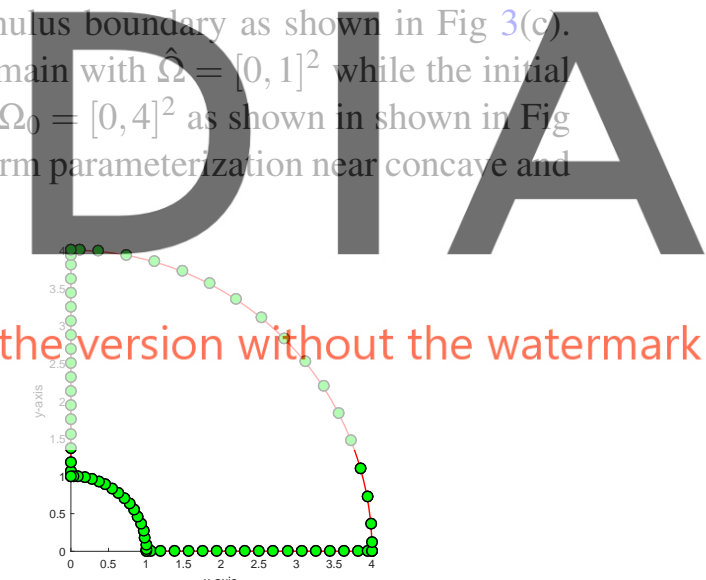

(c) $\partial \Omega_{1}$

Figure 3: Initial parameterization of the computational domain using $p=q=3$ uniform $k$-multiple end knots and with $n_{\xi} \times n_{\eta}=361$ control points consistent with the given boundary curves for the annulus geometry: (a) parametric domain with knot lines and collocation points; (b) computational domain with control points; and (c) input concave boundary with control points.

convex boundaries (see Fig. Fig 4(d)-(f)), while the Coon's mesh produces a uniform parameterization (see Fig. Fig 4(a)-(c)). However, the Jacobian is satisfied in both of the parameterization as shown in Fig 4. By further applying a position constraint in fixing the center control point at the center position of the physical domain a uniform and regular parameterization is also achieved using the IGA-C-PDE 
method (see Fig. Fig 4(g)-(i)). For constrained IGA-C-PDE as discussed in the previous section, a least squares solution is obtained while exactly meeting all constraints, including the boundary curves and the center position of the physical domain. This constraint can be very useful for constructing a clustering of control points at positions of the physical domain for achieving solution dependent parameterization.

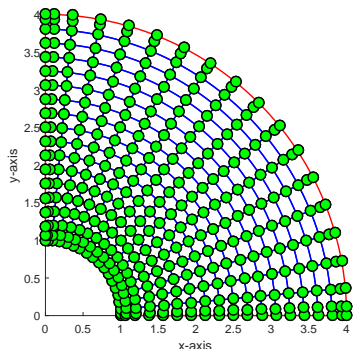

(a)
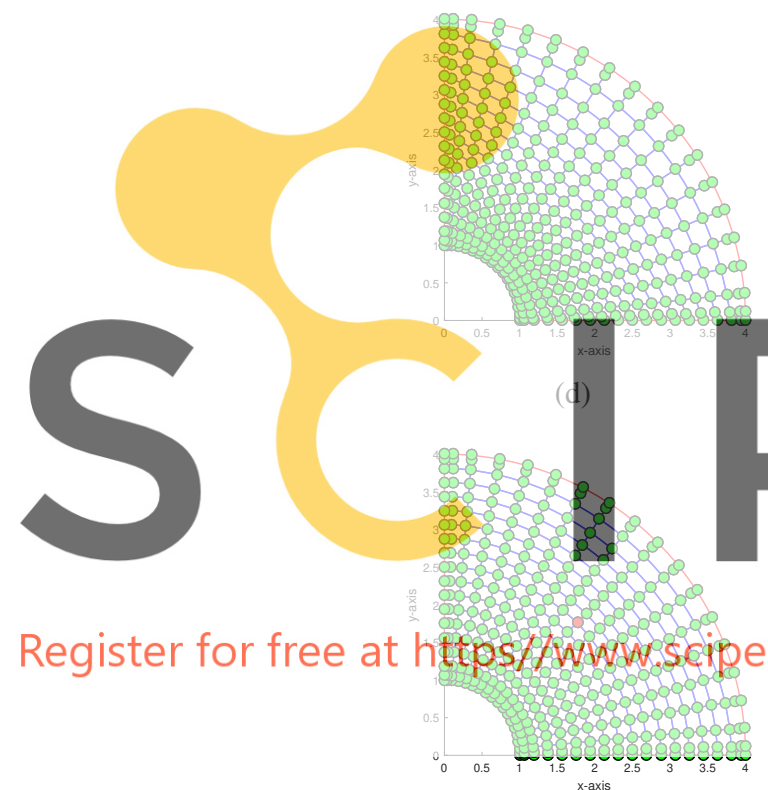

(g)

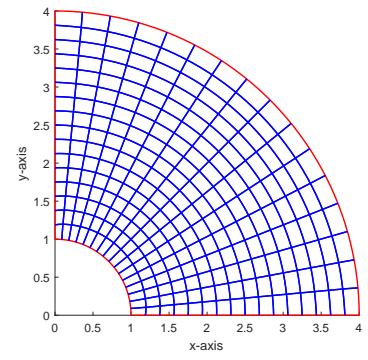

(b)
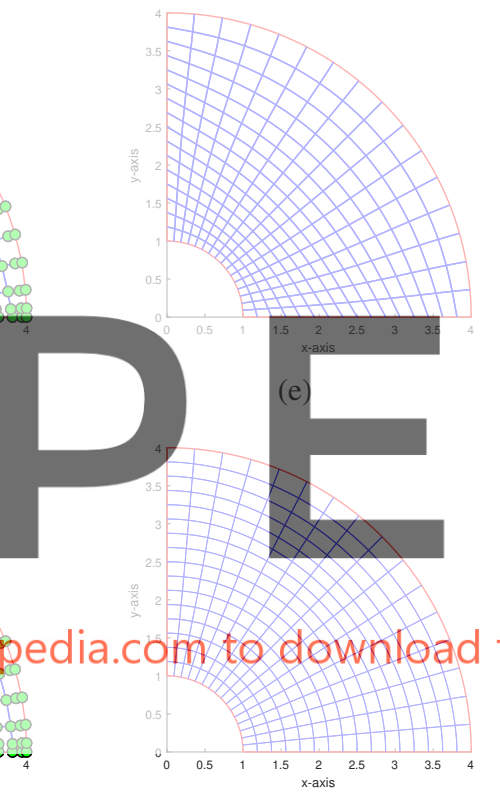

(h)

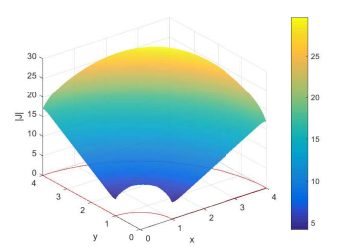

(c)
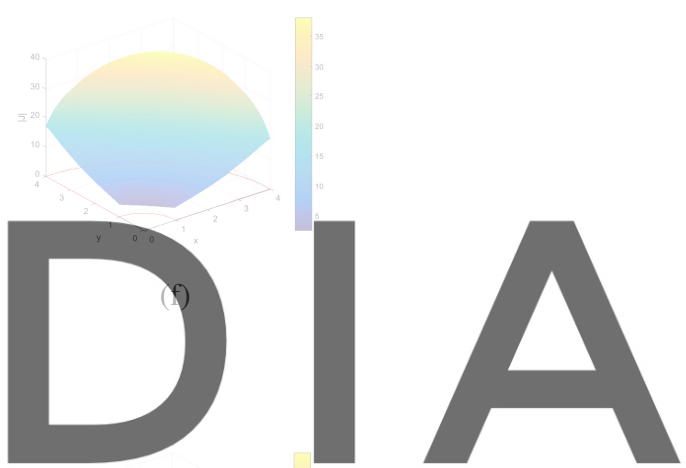

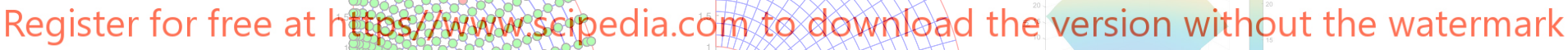

Figure 4: Resulting parameterization for the annulus geometry using Coon's method and our method based on IGA-C-PDE: (a)-(c) parameterization using the Coon's method; (d)-(f) parameterization using the unconstrained IGA-C-PDE method; (g)-(i) parameterization using the IGA-C-PDE method with center position constraints; (left column) parameterization with control points; (middle column) parameterization with parametric curves on the surface at knot lines; and (right column) Jacobian distribution over resulting computational domain.

\subsection{An example for controlling the local DOFs using a ratio constraint}

The distance ratio of control points is a constraint that can be useful to control the density of the control points in a local region. When $\lambda=0.5$, the resulting parameterization will be uniformly distributed. An example in this case is first shown in Fig. 5 using a square computational domain for the three schemes 
discussed in the previous section using an uniform $\lambda=0.5$ ratio. Smooth and uniform parameterization is achieved in such cases. In this and other examples in this subsection with $\lambda$-ratio control, the initial parameterization of the computational domain is defined with $(\xi, \eta) \in \hat{\Omega}=[0,1]^{2}$ using $p=q=3$ with uniform $k$-multiple end knots and with $n_{\xi} \times n_{\eta}=361$ control points consistent with the given boundary curves for the square domain: The initial computational domain $(x, y)$ is defined as a simple square domain $\Omega_{0}=[0,6]^{2}$, while the final parameterization is defined for a square domain as well with $\Omega_{1}=$ $[0,3]^{2}$.

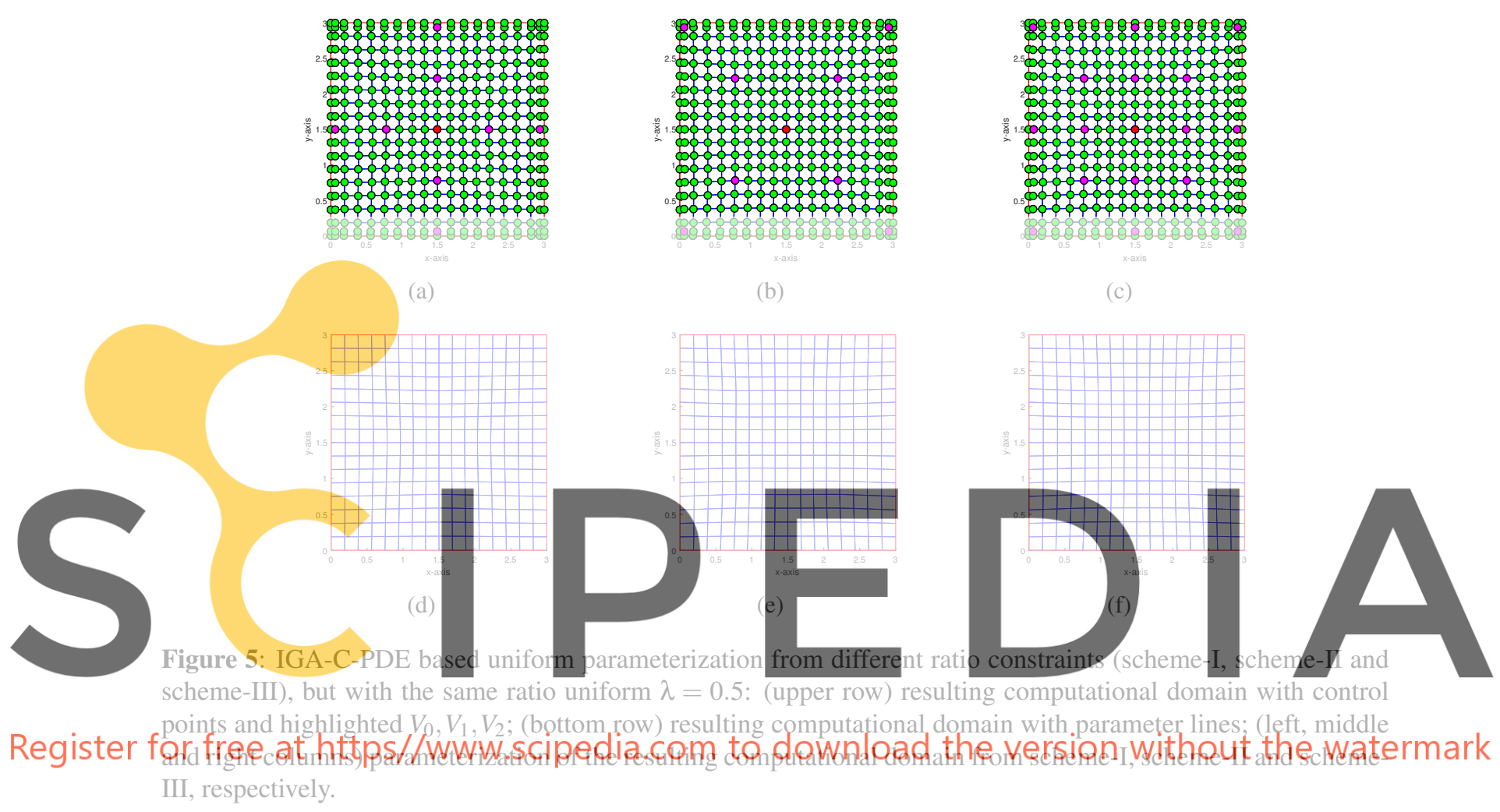

The distribution of the inner control points can be controlled using the $\lambda$ ratio parameter and we can purposefully control the degrees of freedom in a local region of $V_{0}$ for IGA with either higher or lower density as shown in Fig. 6. Combined with the position constraints, one can achieve centralized or decentralized and local clustered distribution of control points, i.e., with either higher density or lower density in terms of the local degrees of freedom for isogeometric analysis in any region centered at $V_{0}$. In addition, by selecting the distance of from $V_{1}, V_{2}$ with reference to $V_{0}$, the size of the region for achieving either higher or lower density of DOFs for IGA can also be controlled. One example with different position constraints for $V_{0}$, but with the same $\lambda$-ration is shown in Fig. 7.

\section{Application for thermal conduction analysis}

The advantage of the new constraints that are introduced along with the elliptical PDE parameterization provides the flexibility in manipulating the parameterization in a desired manner. The constrained IGAC-PDE method is thus particularly useful for constructing solution dependent parameterization. In the 


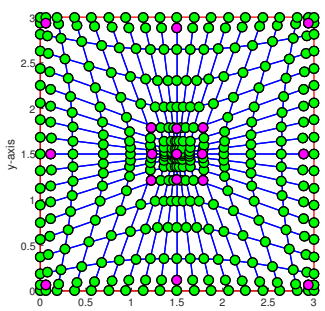

(a)

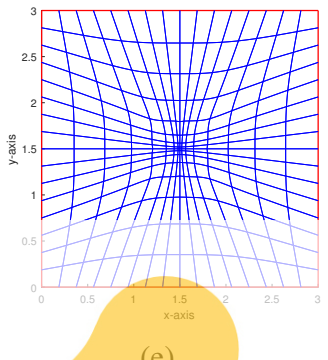

(e)

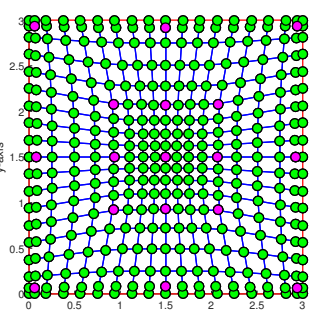

(b)

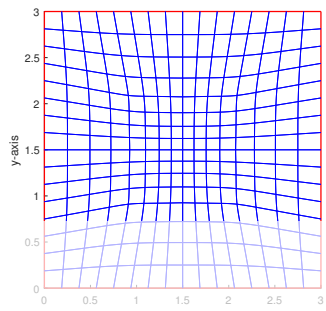

(f)

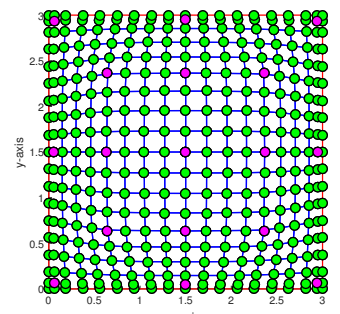

(c)

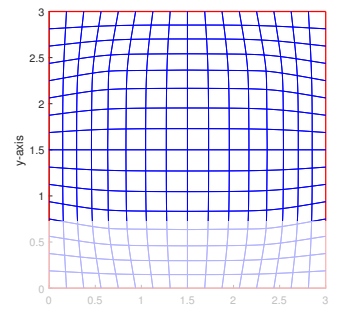

(g)

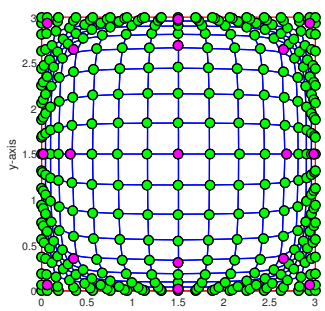

(d)

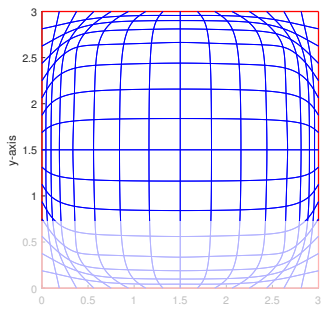

(h)

Figure 6: IGA-C-PDE based parameterization with different ratio $\lambda$ constraints and with free $V_{0}$ : (top and middle rows) resulting parameterization of the computational domain with control points and knot lines display, respectively; (columns from left to right) resulting parameterization with ratio constraints at $\lambda=0.2, \lambda=0.4, \lambda=0.6$

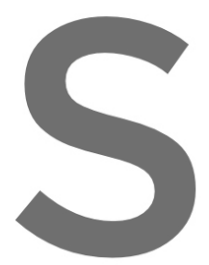
and $\lambda=0.8$, respectively
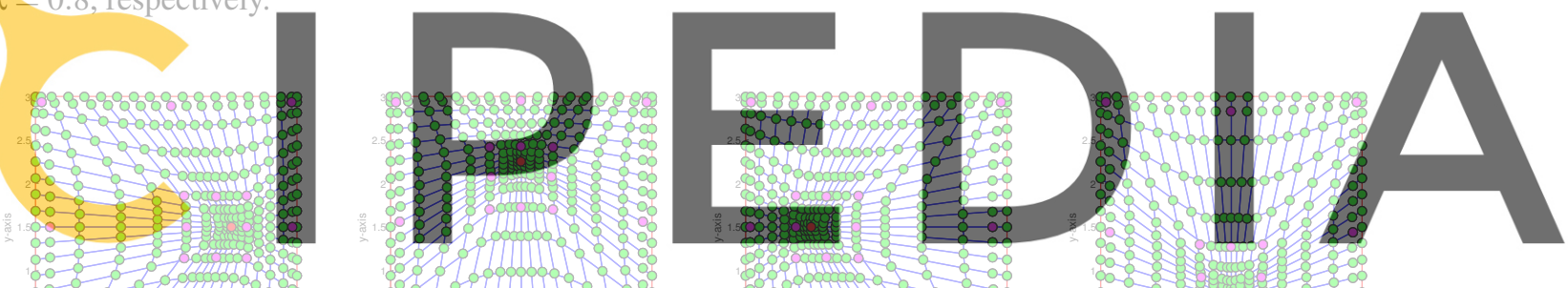

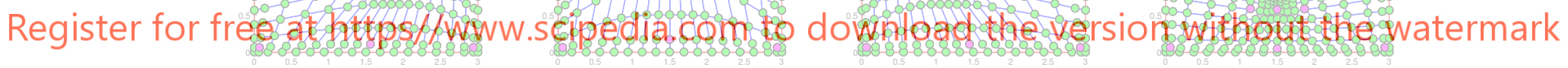

(a)

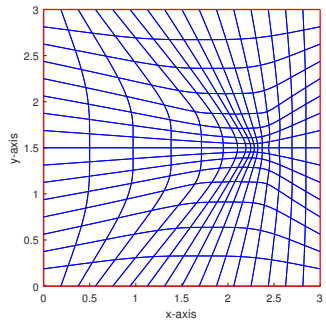

(e) (b)

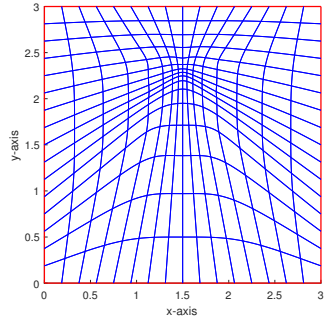

(f) (c)

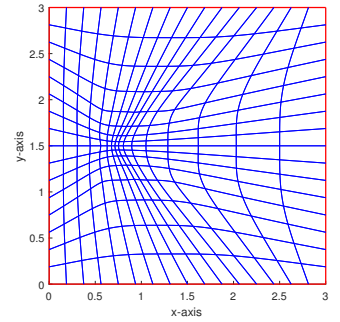

(g) (d)

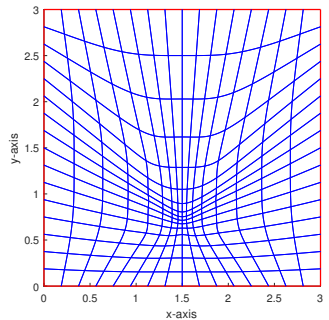

(h)

Figure 7: IGA-C-PDE based parameterization with fixed ratio $\lambda=0.25$ constraints and with different position constraints applied at $V_{0}$ : (top and middle rows) resulting parameterization of the computational domain with control points and knot lines display, respectively; (columns from left to right) resulting parameterization with different position constraints at $V_{0}=(2.25,1.5), V_{0}=(1.5,2.25), V_{0}=(0.75,1.5)$ and $V_{0}=(1.5,0.75)$, respectively. 
following, we show an examples in using the resulting parameterization for thermal conduction analysis. Given a physical domain enclosed by boundary $\Omega=\Omega_{D} \cup \Omega_{N}$, we consider the following thermal conduction problem

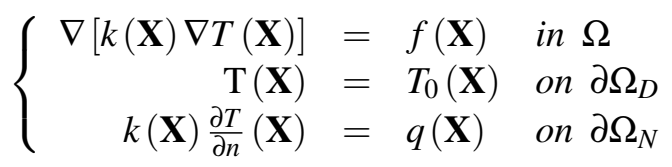

where $\mathbf{X}$ stands for Cartesian coordinates, $\mathrm{T}$ represents the temperature field, $\mathrm{k}$ is the thermal conductivity, $T_{0}$ specifies the imposed temperature on boundary $\partial \Omega_{D}$, q is the thermal flux, and $\nabla T$ is the temperature gradient with $\nabla=\frac{\partial}{\partial \mathbf{X}}$. In order to see the importance of solution dependent parameteriza-

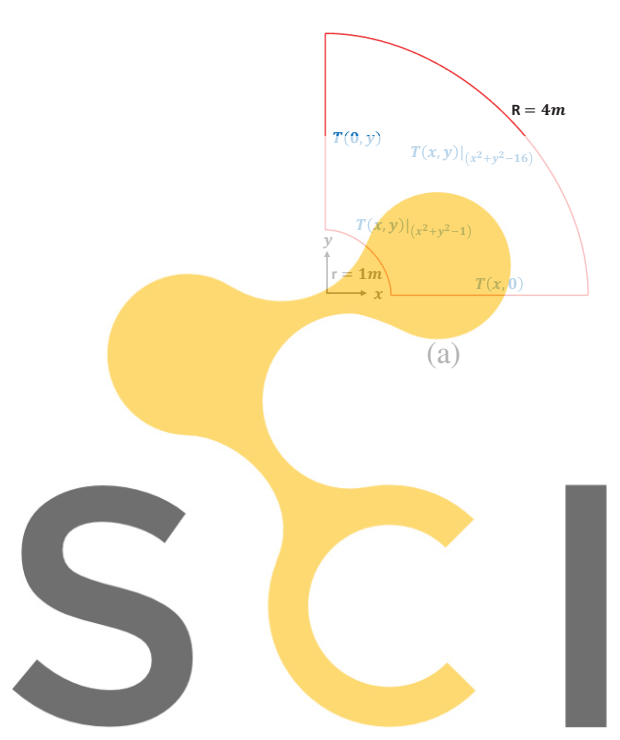

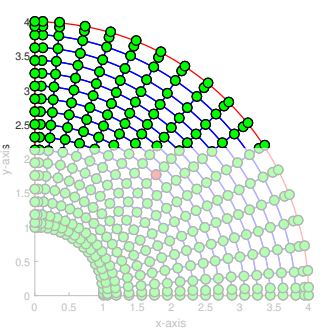

(b)

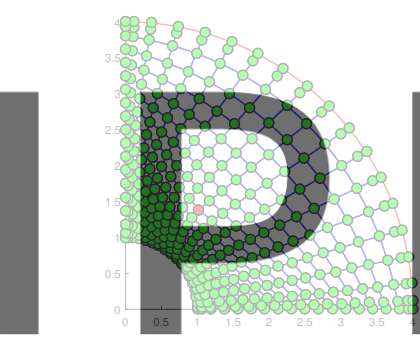

(e)

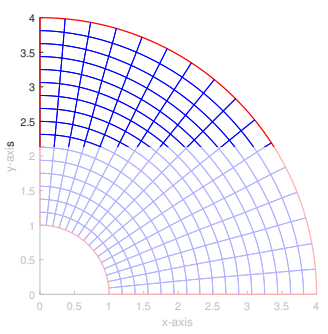

(c)

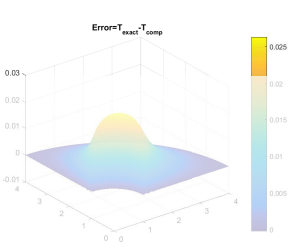

(d)

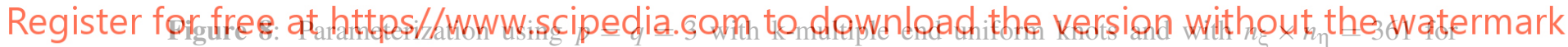

heat conduction test: (a) boundary conditions with $T(x, y)=\frac{1}{x+y} \cdot e^{\frac{1}{x+y}}$; (b)-(d) solution using IGA-C-PDE based parameterization-I, a uniform reguiar parameterization; (e)-(g) solution using IGA-C-PDE based parameterizationII, a parameterization with purposefully shifted control vertices; (left and middle columns) the respective resulting computational domain; and (right column) the respective solution errors.

tion, we assume an annulus physical domain with quarter circular shape represented by a cubic B-spline surface. We have purposefully designed a temperature distribution function as the theoretical solution of the thermal conduction problem and the temperature function has highly varying local region near the inner circular edge of the annulus domain.

$$
T(x, y)=\frac{1}{x+y} \cdot e^{\frac{1}{x+y}}
$$

Two parameterizations as shown in Fig. 17 are used for solving the heat conduction problem with exact solution given in Eq. (18) over the Annulus domain $[0,4] \times[0,4]$. Parameterization-I is a regular and uniform mesh while parameterization-II is motivated from the solution and design accordingly. 
The solution is also produced using isogeometric collocation (IGA-C) method. As shown in Fig. 17, parameterization-II achieves a better and more accurate solution compared with that of parameterizationI. Although, parameterization-I is more uniform but for this particular problem we find that cluster of control points near the inner circular curve produces better solution with less error.

\section{Conclusions}

In this study, we apply the idea of PDE-based grid generation in FEA for analysis suitable (AS) parameterization in IGA based on isogeometric collocation (IGA-C) methods. Given an arbitrary boundary, the Laplace PDE is applied to produce a smooth parameterization of the computational domain defined by th input boundary for IGA applications. The PDE for parameterization is also solved using IGA$\mathrm{C}$ methods with input boundary geometry as Dirichlet conditions and with inner control points of the computational domain as unknowns of the PDE. Additional position and ratio constraints along with the arbitrary input domain boundary have also been introduced in order to produce solution-dependent parameterization. With the present constrained IGA-C-PDE methods, B-splines/NURBS are used for parameterization and the solution can be efficiently solved using constrained linear least squares solutions, which is more efficient compared with most other know methods using non-linear optimization for analysis suitable parameterization for IGA. In our future work, the IGA-C-PDE method can also been extended for parameterization using other modeling schemes with complex domain boundaries.

\section{Acknowledgment}

The work described in this paper was partially supported by the Research Grants Council of the Hong Kong Special Administrative Region, China (GRF Grants Nos. CityU11201919 and CityU11206917).

\section{REFERENCES}

[1] T. J. Hughes, J. A. Cottrell, Y. Bazilevs, Isogeometric analysis: Cad, finite elements, Nurbs, exact geometry and mesh refinement, Computer methods in applied mechanics and engineering 194 (39) (2005) 4135-4195. doi:10.1016/j.cma.2004.10.008.

[2] F. Auricchio, L. B. Da Veiga, T. Hughes, A. Reali, G. Sangalli, Isogeometric collocation methods, Mathematical Models and Methods in Applied Sciences 20 (11) (2010) 2075-2107. doi:10.1142/ S0218202510004878.

[3] P. Fedeli, A. Frangi, F. Auricchio, A. Reali, Phase-field modeling for polarization evolution in ferroelectric materials via an isogeometric collocation method, Computer Methods in Applied Mechanics and Engineering 351 (2019) 789-807. doi:10.1016/j.cma.2019.04.001.

[4] R. Kruse, N. Nguyen-Thanh, L. De Lorenzis, T. Hughes, Isogeometric collocation for large deformation elasticity and frictional contact problems, Computer Methods in Applied Mechanics and Engineering 296 (2015) 73-112. doi:10.1016/j.cma.2015.07.022.

[5] Z. Ali, W. Ma, Isogeometric collocation method with intuitive derivative constraints for pde-based analysis-suitable parameterization, Computer Aided Geometric Design (under review) (2021).

[6] G. Xu, B. Mourrain, R. Duvigneau, A. Galligo, Constructing analysis-suitable parameterization of computational domain from cad boundary by variational harmonic method, Journal of Computational Physics 252 (2013) 275-289. doi:10.1016/j. jcp.2013.06.029. 
[7] S. Gondegaon, H. K. Voruganti, An efficient parametrization of planar domain for isogeometric analysis using harmonic functions, Journal of the Brazilian Society of Mechanical Sciences and Engineering 40 (10) (2018) 493. doi:10.1007/s40430-018-1414-z.

[8] G. Xu, M. Li, B. Mourrain, T. Rabczuk, J. Xu, S. P. Bordas, Constructing iga-suitable planar parameterization from complex cad boundary by domain partition and global/local optimization, Computer Methods in Applied Mechanics and Engineering 328 (2018) 175-200. doi:10.1016/ j.cma.2017.08.052.

[9] G. Xu, B. Mourrain, R. Duvigneau, A. Galligo, Optimal analysis-aware parameterization of computational domain in isogeometric analysis, in: International Conference on Geometric Modeling and Processing, Springer, 2010, pp. 236-254.

[10] G. Xu, B. Mourrain, R. Duvigneau, A. Galligo, Parameterization of computational domain in isogeometric analysis: methods and comparison, Computer Methods in Applied Mechanics and Engineering 200 (23) (2011) 2021-2031. doi:10.1016/j.cma.2011.03.005.

[11] T. Martin, E. Cohen, M. Kirby, Volumetric parameterization and trivariate b-spline fitting using harmonic functions, in: Proceedings of the 2008 ACM symposium on Solid and physical modeling, ACM, 2008, pp. 269-280. doi:10.1016/j.cagd.2008.09.008.

[12] J. Gravesen, A. Evgrafov, D.-M. Nguyen, P. Nørtoft, Planar parametrization in isogeometric analysis, in: International Conference on Mathematical Methods for Curves and Surfaces, Springer, 2012, pp. 189-212. doi:10.1007/978-3-642-54382-1_11.

[13] A. Falini, J. Špeh, B. Jüttler, Planar domain parameterization with thb-splines, Computer Aided Geometric Design 35 (2015) 95-108. doi:10.1016/j.cagd.2015.03.014.

[14] S. P. Spekreijse, Elliptic grid generation based on laplace equations and algebraic transformations, Journal of Computational Physics 118 (1) (1995) 38-61. doi:10.1006/ jcph.1995.1078.

[15] H. Lin, Y. Xiong, X. Wang, Q. Hu, J. Ren, Isogeometric least-squares collocation method with consistency and convergence analysis, Journal of Systems Science and Complexity 33 (5) (2020) 1656-1693. doi:https://doi.org/10.1007/s11424-020-9052-9. 